\title{
The First Hochschild Cohomology of Square Algebras With it's Stability
}

\author{
Feysal Hassani ${ }^{1} \&$ Negin Salehi Oroozaki ${ }^{2}$ \\ ${ }^{1}$ Department of Mathematics, Payame Noor University, Tehran, Iran \\ 2 Department of Mathematics, Payame Noor University, Tehran, Iran \\ Correspondence: Feysal Hassani, Department of Mathematics, Payame Noor University, Tehran, Iran. \\ E-mail: feysal.hassani.pnu@gmail.com
}

Received: April 18, 2017 Accepted: May 8, 2017 Online Published: July 15, 2017

doi:10.5539/jmr.v9n4p200 URL: https://doi.org/10.5539/jmr.v9n4p200

\begin{abstract}
In this paper, we study on a special case of generalized matrix algebra that we call it square algebra. According to that Hochschild cohomology play a significant role in Geometry for example in orbifolds, we study the first Hochschild cohomology of the square algebra the vanishing of its.
\end{abstract}

Keywords: First Hochschild cohomology, Hochschild cohomology, Square algebra

\section{Introduction}

Let $\mathcal{R}$ be a commutative ring (with unit), let $A$ and $B$ be $\mathcal{R}$-algebras and $M$ be a left $A$-module and right $B$-module. A triangular algebra $T$ over $\mathcal{R}$ is the following matrix

$$
T=\left[\begin{array}{cc}
A & M \\
& B
\end{array}\right] .
$$

Automorphisms, derivations, commuting mappings and Lie derivations on triangular algebras are studied by Cheung (Cheung, 2001) and (Cheung, 2003). Other useful and valuable literature concerning the structure of derivations and Lie derivations is ( $\mathrm{Ji} \& \mathrm{Qi}, 2011)$. Basic examples of triangular algebras are upper triangular matrix algebras and nest algebras which derivations of those considered in (Christensen, 1977), (Coelho, \& Milies, 1993), (Donsig, Forrest \& Marcoux, 1996).

A generalized matrix algebra is a generalization of triangular matrix algebra. In the triangular algebra $\mathcal{T}$, the element lies in the second row and second column is zero. In generalized matrix algebra, we put a right $A$-module and left $B$-module $N$ in zero place. We denote the generalized matrix algebra by $\mathcal{G}$. Algebraic studying on derivations, generalized derivations and Lie derivations have been studied in (Du, \& Wang, 2012), (Li \& Wei, 2012), (Li, \& Xiao, 2011).

Throughout this paper $\mathcal{R}$ is a commutative ring (with unit), $A$ and $B$ are $R$-algebras with units $1_{A}$ and $1_{B}$, respectively, $M$ is an $\mathcal{R}$-bimodule, left $A$-module and right $B$-module $(A, B$-module) and $N$ is an $\mathcal{R}$-bimodule, right $A$-module and left $B$-module $\left(B, A\right.$-module). Define bimodule homomorphisms $\Phi_{M N}: M \otimes_{B} N \longrightarrow A$ and $\Phi_{N M}: N \otimes_{A} M \longrightarrow B$ satisfying the following commutative diagrams:

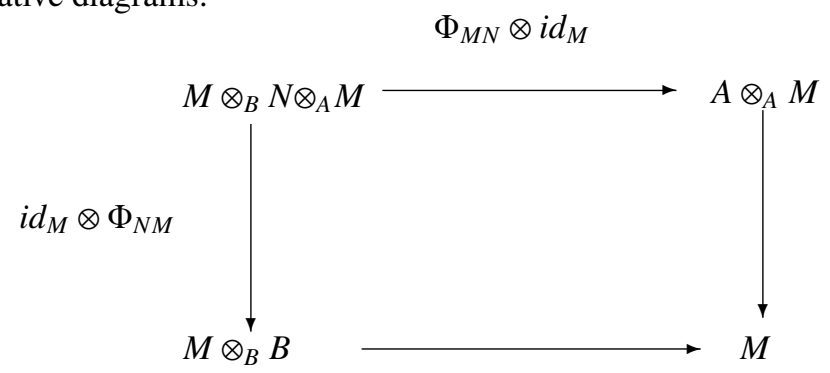


and

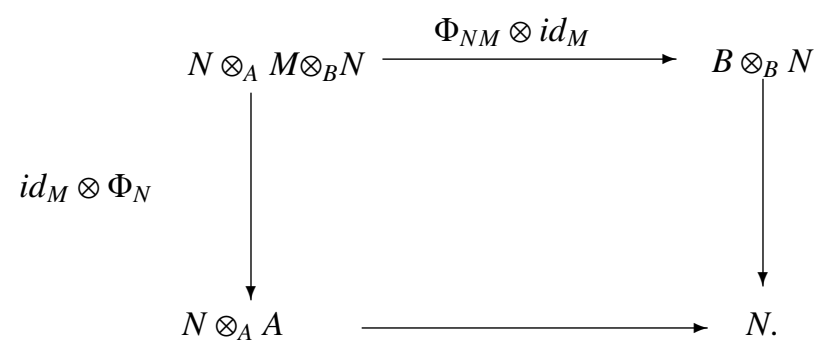

For more details and applications see (Buchweitz, 2003). We define generalized matrix algebra

$$
\mathcal{G}=\left[\begin{array}{cc}
A & M \\
N & B
\end{array}\right]=\left\{\left[\begin{array}{cc}
a & m \\
n & b
\end{array}\right]: a \in A, b \in B, m \in M, n \in N\right\},
$$

with the usual $2 \times 2$ matrix-like addition and multiplication

$$
\left[\begin{array}{cc}
a_{1} & m_{1} \\
n_{1} & b_{1}
\end{array}\right] \cdot\left[\begin{array}{cc}
a_{2} & m_{2} \\
n_{2} & b_{2}
\end{array}\right]=\left[\begin{array}{cc}
a_{1} a_{2}+m_{1} \otimes_{B} n_{2} & a_{1} m_{2}+m_{1} b_{2} \\
n_{1} a_{2}+b_{1} n_{2} & n_{1} \otimes_{A} m_{2}+b_{1} b_{2}
\end{array}\right]
$$

In this algebra, if $M \otimes_{B} N=0=N \otimes_{A} M$, then we denote it by $S$ and we called that a square algebra.

Let $\mathcal{R}$ be a commutative ring (with unit), let $A$ be an $\mathcal{R}$-algebra and $M$ be an $A$-bimodule. For $n=0,1,2, \ldots$, let $C^{n}(A, M$ ) be the space of all n-linear (as a $\mathcal{R}$-module map) mappings from $A \times \cdots \times A$ into $M$ and $C^{0}(A, M)=M$. Consider the sequence

$$
0 \longrightarrow C^{0}(A, M) \stackrel{d^{0}}{\longrightarrow} C^{1}(A, M) \stackrel{d^{1}}{\longrightarrow} \cdots(\tilde{C}(A, M))
$$

in which

$$
\begin{aligned}
d^{0} x(a)= & a x-x a \\
d^{n} f\left(a_{1}, a_{2}, \cdots, a_{n+1}\right)= & a_{1} f\left(a_{2}, \cdots, a_{n+1}\right) \\
& +(-1)^{n+1} f\left(a_{1}, \cdots, a_{n}\right) a_{n+1} \\
& +\sum_{j=1}^{n}(-1)^{j} f\left(a_{1}, \cdots, a_{j-1}, a_{j} a_{j+1}, \cdots, a_{n+1}\right)
\end{aligned}
$$

where $n \geq 1, x \in M$ and $a_{1}, \ldots, a_{n+1} \in A$. The above sequence is a complex for $A$ and $M$. The $\mathrm{n}$-th cohomology group of $\tilde{C}(A, E)$ is said to be n-th Hochschild cohomology group and denoted by $H^{n}(A, M)$, for more details see (Brodmann \& Sharp, 1998), (Rotman, 2009). A derivation is a linear map $D: A \longrightarrow M$ such that $D(a b)=a D(b)+D(a) b(a, b \in A)$ and for $x \in M$, we define the map $D_{x}: A \longrightarrow M$ by $D_{x}(a)=x a-a x$. The map $D_{x}$ is a derivation and such derivations called inner derivations. Let $\operatorname{Der}(A, M)$ denote all derivations and $\operatorname{Inn}(A, M)$ denote all inner derivations.

Thus, we have

$$
H^{1}(A, M)=\frac{\operatorname{Der}(A, M)}{\operatorname{Inn}(A, M)} .
$$

In this paper, we describe $H^{1}(S, S)$ and vanishing of $H^{1}(S, X)$, where $X$ is a two sided $S$-module (bimodule) is investigated.

\section{Structure of $H^{1}(S, S)$}

We begin with the following simple properties of derivations on $S$ as follows:

Proposition 1 Let $D: S \longrightarrow S$ be a derivation, then there are derivations $d_{A}: A \longrightarrow A, d_{B}: B \longrightarrow B$, $\mathcal{R}$-linear maps $\tau: M \longrightarrow M$ and $\sigma: N \longrightarrow N$ and elements $m_{D} \in M$ and $n_{D} \in N$ such that

(i) $D\left(\left[\begin{array}{cc}1_{A} & 0 \\ 0 & 0\end{array}\right]\right)=\left[\begin{array}{cc}0 & m_{D} \\ n_{D} & 0\end{array}\right]=-D\left(\left[\begin{array}{cc}0 & 0 \\ 0 & 1_{B}\end{array}\right]\right)$,

(ii) $D\left(\left[\begin{array}{cc}0 & m \\ 0 & 0\end{array}\right]\right)=\left[\begin{array}{cc}0 & \tau(m) \\ 0 & 0\end{array}\right]$ and $D\left(\left[\begin{array}{ll}0 & 0 \\ n & 0\end{array}\right]\right)=\left[\begin{array}{cc}0 & 0 \\ \sigma(n) & 0\end{array}\right]$, 
(iii) $D\left(\left[\begin{array}{ll}a & 0 \\ 0 & 0\end{array}\right]\right)=\left[\begin{array}{cc}d_{A}(a) & a m_{D} \\ n_{D} a & 0\end{array}\right]$ and $D\left(\left[\begin{array}{ll}0 & 0 \\ 0 & b\end{array}\right]\right)=\left[\begin{array}{cc}0 & -m_{D} b \\ -b n_{D} & d_{B}(b)\end{array}\right]$,

(iv) $\tau\left(\right.$ am) $=d_{A}(a) m+a \tau(m)$,

(v) $\tau(m b)=\tau(m) b+m d_{B}(b)$

(vi) $\sigma(n a)=n d_{A}(a)+\sigma(n) a$,

(vii) $\sigma(b n)=b \sigma(n)+d_{B}(b) n$,

for all $a \in A, b \in B, m \in M, n \in N$.

Conversely, if $d_{A}$ and $d_{B}$ are derivations on $A$ and $B$, respectively, and if $\tau: M \longrightarrow M$ and $\sigma: N \longrightarrow N$ are any $\mathcal{R}$-linear maps satisfy (i), (ii), (iii) and (iv) then the map

$$
D\left(\left[\begin{array}{cc}
a & m \\
n & b
\end{array}\right]\right)=\left[\begin{array}{cc}
d_{A}(a) & \tau(m) \\
\sigma(n) & d_{B}(b)
\end{array}\right]
$$

defines a derivation on $S$.

Proof. Let $D$ be a derivation. By the following relations and simple calculation we obtain (i)-(vii):

$$
D\left(\left[\begin{array}{cc}
0 & a m \\
0 & 0
\end{array}\right]\right)=D\left(\left[\begin{array}{cc}
a & 0 \\
0 & 0
\end{array}\right]\left[\begin{array}{cc}
0 & m \\
0 & 0
\end{array}\right]\right), D\left(\left[\begin{array}{cc}
0 & m b \\
0 & 0
\end{array}\right]\right)=D\left(\left[\begin{array}{cc}
0 & m \\
0 & 0
\end{array}\right]\left[\begin{array}{cc}
0 & 0 \\
0 & b
\end{array}\right]\right),
$$

and

$$
D\left(\left[\begin{array}{cc}
0 & 0 \\
b n & 0
\end{array}\right]\right)=D\left(\left[\begin{array}{ll}
0 & 0 \\
0 & b
\end{array}\right]\left[\begin{array}{cc}
0 & 0 \\
n & 0
\end{array}\right]\right), D\left(\left[\begin{array}{cc}
0 & 0 \\
n a & 0
\end{array}\right]\right)=D\left(\left[\begin{array}{cc}
0 & 0 \\
n & 0
\end{array}\right]\left[\begin{array}{ll}
a & 0 \\
0 & 0
\end{array}\right]\right) .
$$

Conversely, consider,

$$
\begin{aligned}
D\left(\left[\begin{array}{cc}
a_{1} & m_{1} \\
n_{1} & b_{1}
\end{array}\right]\left[\begin{array}{cc}
a_{2} & m_{2} \\
n_{2} & b_{2}
\end{array}\right]\right) & =D\left(\left[\begin{array}{cc}
a_{1} a_{2} & a_{1} m_{2}+m_{1} b_{2} \\
n_{1} a_{1}+b_{1} n_{2} & b_{1} b_{2}
\end{array}\right]\right) \\
& =\left[\begin{array}{cc}
d_{A}\left(a_{1} a_{2}\right) & \tau\left(a_{1} m_{2}+m_{1} b_{2}\right) \\
\sigma\left(n_{1} a_{1}+b_{1} n_{2}\right) & d_{B}\left(b_{1} b_{2}\right) .
\end{array}\right]
\end{aligned}
$$

Moreover,

$$
\begin{aligned}
{\left[\begin{array}{cc}
a_{1} & m_{1} \\
n_{1} & b_{1}
\end{array}\right] D\left(\left[\begin{array}{cc}
a_{2} & m_{2} \\
n_{2} & b_{2}
\end{array}\right]\right)+D\left(\left[\begin{array}{cc}
a_{1} & m_{1} \\
n_{1} & b_{1}
\end{array}\right]\right)\left[\begin{array}{ll}
a_{2} & m_{2} \\
n_{2} & b_{2}
\end{array}\right]=} & {\left[\begin{array}{cc}
a_{1} d_{A}\left(a_{2}\right) & a_{1} \tau\left(m_{2}\right)+m_{1} d_{B}\left(b_{2}\right) \\
n_{1} d_{A}\left(a_{2}\right)+b_{1} \sigma\left(n_{2}\right) & b_{1} d_{B}\left(b_{2}\right)
\end{array}\right] } \\
& +\left[\begin{array}{cc}
d_{A}\left(a_{1}\right) a_{2} & d_{A}\left(a_{1}\right) m_{2}+\tau\left(m_{1}\right) b_{2} \\
\sigma\left(n_{1}\right) a_{2}+d_{B}\left(b_{1}\right) n_{2} & d_{B}\left(b_{1}\right) b_{2}
\end{array}\right] \\
= & {\left[\begin{array}{cc}
a_{1} d_{A}\left(a_{2}\right)+d_{A}\left(a_{1}\right) a_{2} & a_{1} \tau\left(m_{2}\right)+m_{1} d_{B}\left(b_{2}\right) \\
n_{1} d_{A}\left(a_{2}\right)+b_{1} \sigma\left(n_{2}\right) & +d_{A}\left(a_{1}\right) m_{2}+\tau\left(m_{1}\right) b_{2} \\
\sigma\left(n_{1}\right) a_{2}+d_{B}\left(b_{1}\right) n_{2} & b_{1} d_{B}\left(b_{2}\right)+d_{B}\left(b_{1}\right) b_{2}
\end{array}\right] } \\
= & {\left[\begin{array}{cc}
d_{A}\left(a_{1} a_{2}\right) & \tau\left(a_{1} m_{2}\right)+\tau\left(m_{1} b_{2}\right) \\
\sigma\left(n_{1} a_{2}\right)+\sigma\left(b_{1} n_{2}\right) & d_{B}\left(b_{1} b_{2}\right)
\end{array}\right] }
\end{aligned}
$$

Thus $D$ is a derivation on $S$.

Let $a_{0} \in A$ and $b_{0} \in B$, then Rosenblum $\mathcal{R}$-linear map $\tau_{M}^{a_{0}, b_{0}}: M \longrightarrow M$ is defined by

$$
\tau_{M}^{a_{0}, b_{0}}(m)=a_{0} \cdot m-m \cdot b_{0} \text { for each } m \in M .
$$

Now, let $Z(A)$ be the center of $A$ and $Z(B)$ be the center of $B, x \in Z(A)$ and $y \in Z(B)$. Then the Rosenblum $\mathcal{R}$-linear map $\tau_{M}^{x, y}$ is called a central Rosenblum $\mathcal{R}$-linear map. We denote the set of all central Rosenblum $\mathcal{R}$-linear maps by $Z R_{A, B}(M)$. Also, we have

$$
Z R_{A, B}(M) \subseteq \operatorname{Hom}_{A, B}(M) .
$$


An $\mathcal{R}$-map $\tau_{M}: M \longrightarrow M$ is called a generalized Rosenblum $\mathcal{R}$-linear map if there exist derivations $d_{A}$ and $d_{B}$ on $A$ and $B$, respectively, such that $\tau_{M}$ satisfies

$$
\tau(a m b)=d_{A}(a) m b+a \tau(m) b+a m d_{B}(b)
$$

for each $a \in A, b \in B$ and $m \in M$. Similarly, an $\mathcal{R}$-map $\tau_{N}: N \longrightarrow N$ is called a generalized Rosenblum $\mathcal{R}$-linear map if there exist derivations $d_{A}$ and $d_{B}$ on $A$ and $B$, respectively, such that $\tau_{N}$ satisfies

$$
\tau(b n a)=d_{B}(b) n a+b \tau_{N}(n) a+b n d_{A}(a)
$$

for each $a \in A, b \in B$ and $m \in M$.

Lemma 2 Let $\varphi \in \operatorname{Hom}_{A, B}(M)$ and $\sigma \in \operatorname{Hom}_{B, A}(N)$. Then the map $d_{\varphi, \sigma}: S \longrightarrow S$ given by

$$
d_{\varphi, \sigma}\left(\left[\begin{array}{cc}
a & m \\
n & b
\end{array}\right]\right)=\left[\begin{array}{cc}
0 & \varphi(m) \\
\sigma(n) & 0
\end{array}\right]
$$

is a derivation. Moreover, $d_{\varphi, \sigma}$ is an inner derivation if and only if $\varphi=\tau_{M}^{x, y}$ and $\sigma=\tau_{N}^{y, x}$, where $\tau_{M}^{x, y} \in Z R_{A, B}(M)$ and $\tau_{N}^{y, x} \in Z R_{B, A}(N)$.

Proof. The first statement follows immediately from assume that $\varphi=\tau_{M}^{x, y}$ and $\sigma=\tau_{N}^{y, x}$ where $x \in Z(A)$ and $y \in Z(B)$. Then

$$
\begin{aligned}
& d_{\left[\begin{array}{ll}
x & 0 \\
0 & y
\end{array}\right]}\left(\left[\begin{array}{ll}
a & m \\
n & b
\end{array}\right]\right)=\left[\begin{array}{cc}
x & 0 \\
0 & y
\end{array}\right]\left[\begin{array}{ll}
a & m \\
n & b
\end{array}\right]-\left[\begin{array}{ll}
a & m \\
n & b
\end{array}\right]\left[\begin{array}{ll}
x & 0 \\
0 & y
\end{array}\right] \\
& =\left[\begin{array}{cc}
x a & x m \\
y n & y b
\end{array}\right]-\left[\begin{array}{cc}
a x & m y \\
n x & b y
\end{array}\right] \\
& =\left[\begin{array}{cc}
x a-a x & x m-m y \\
y n-n x & y n-b y
\end{array}\right]=\left[\begin{array}{cc}
0 & x m-m y \\
y n-n x & 0
\end{array}\right] \\
& =\left[\begin{array}{cc}
0 & \varphi(m) \\
\sigma(n) & 0
\end{array}\right] \text {. }
\end{aligned}
$$

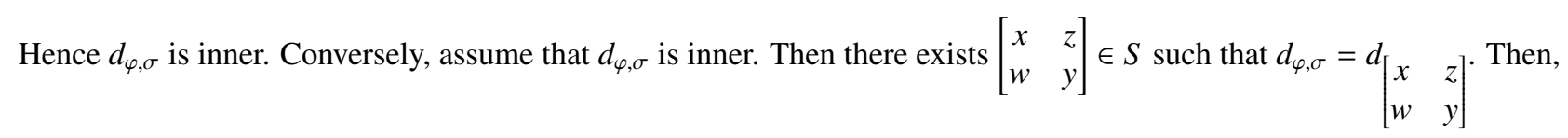

$$
\begin{aligned}
& d_{\left[\begin{array}{ll}
x & z \\
w & y
\end{array}\right]}\left(\left[\begin{array}{ll}
a & m \\
n & b
\end{array}\right]\right)=\left[\begin{array}{cc}
x & z \\
w & y
\end{array}\right]\left[\begin{array}{ll}
a & m \\
n & b
\end{array}\right]-\left[\begin{array}{ll}
a & m \\
n & b
\end{array}\right]\left[\begin{array}{ll}
x & z \\
w & y
\end{array}\right] \\
& =\left[\begin{array}{cc}
x a-a x & x m+z b-a z-m y \\
w a+y n-n x-b w & y b-b y
\end{array}\right] .
\end{aligned}
$$

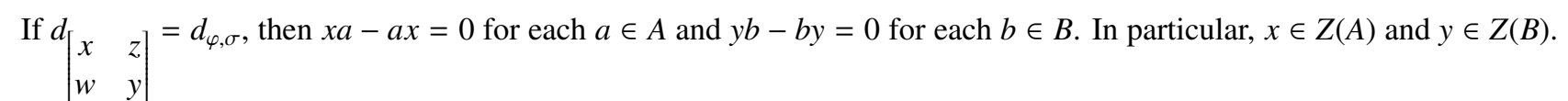

Moreover, we have

$$
\varphi(m)=x m+z b-a z-m y
$$

and

$$
\sigma(n)=w a+y n-n x-b w .
$$

Since $\varphi \in \operatorname{Hom}_{A, B}(M)$ and $\sigma \in \operatorname{Hom}_{B, A}(N)$, it follows that $z b-a z=0$ and $w a-b w=0$. Hence $\varphi(m)=x m-m y=\tau_{M}^{x, y}(m)$ and $\sigma(n)=y n-n x=\tau_{N}^{y, x}(n)$. In particular, $\varphi \in Z R_{A, B}(M)$ and $\sigma \in Z R_{B, A}(N)$.

We can now state the main result of this section for describing $H^{1}(S, S)$.

Theorem 3 If $H^{1}(A, A)=0=H^{1}(B, B)$, then

$$
H^{1}(S, S) \cong \frac{\operatorname{Hom}_{A, B}(M) \times \operatorname{Hom}_{B, A}(N)}{Z R_{A, B}(M) \times Z R_{B, A}(N)} .
$$


Proof. Define $\phi: \operatorname{Hom}_{A, B}(M) \times \operatorname{Hom}_{B, A}(N) \longrightarrow H^{1}(S, S)$ by

$$
\phi(\varphi, \sigma)=\bar{d}_{\varphi, \sigma},
$$

where $\bar{d}_{\varphi, \sigma}$ represents the equivalence class of $d_{\varphi, \sigma}$ in $H^{1}(S, S)$. Clearly, $\phi$ is $\mathcal{R}$-linear.

We shall show that $\phi$ is surjective. Let $d: S \longrightarrow S$ be a derivation. Then there are derivations $d_{A}, d_{B}$, and $\mathcal{R}$-linear maps $\tau: M \longrightarrow M, \sigma: N \longrightarrow N$ and elements $m_{d} \in M, n_{d} \in N$ that satisfy in the conditions (i)-(vii) of Proposition 1. Since $H^{1}(A, A)=H_{1}(B, B)=0$, we can find $x \in A$ and $y \in B$ such that $d_{A}=d_{x}$ and $d_{B}=d_{y}$. Define $d_{0}: S \longrightarrow S$ by

$$
d_{0}\left(\left[\begin{array}{cc}
a & m \\
n & b
\end{array}\right]\right)=\left[\begin{array}{cc}
d_{x}(a) & \tau_{M}^{x, y}(m)+\left(a m_{d}-m_{d} b\right) \\
\tau_{N}^{y, x}(n)+\left(n_{d} a-b n_{d}\right) & d_{y}(b)
\end{array}\right] .
$$

Then $d_{0}$ is an inner derivation on $S$ induced by $\left[\begin{array}{cc}x & -m_{d} \\ -n_{d} & y\end{array}\right]$. Furthermore, if $d_{1}=d-d_{0}$, then $d_{1}$ is a derivation and

$$
\begin{aligned}
d_{1}\left(\left[\begin{array}{cc}
a & m \\
n & b
\end{array}\right]\right) & =\left[\begin{array}{cc}
d_{x}(a) & \tau(m)+\left(a m_{d}-m_{d} b\right) \\
\sigma(n)+\left(n_{d} a-b n_{d}\right) & d_{y}(b)
\end{array}\right] \\
& -\left[\begin{array}{cc}
d_{x}(a) & \tau_{M}^{x, y}(m)+\left(a m_{d}-m_{d} b\right) \\
\tau_{N}^{y, x}(n)+\left(n_{d} a-b n_{d}\right) & d_{y}(b)
\end{array}\right] \\
& =\left[\begin{array}{cc}
0 & \tau(m)-\tau_{M}^{x, y}(m) \\
\sigma(n)-\tau_{N}^{y, x}(n) & 0
\end{array}\right]=\left[\begin{array}{cc}
0 & \tau_{1}(m) \\
\sigma_{1}(n) & 0
\end{array}\right]
\end{aligned}
$$

where $\tau_{1}=\tau-\tau_{M}^{x, y}$ and $\sigma_{1}=\sigma-\tau_{N}^{x, y}$. It follows from Proposition 1, that $\tau_{1} \in \operatorname{Hom}_{A, B}(M)$ and $\sigma_{1} \in H_{o m}(N)$. Finally, set $\bar{d}=\bar{d}_{1}=\varphi\left(\tau_{1}, \sigma_{1}\right)$, and so $\varphi$ is surjective. This implies that

$$
H^{1}(S, S) \cong \frac{\operatorname{Hom}_{A, B}(M) \times \operatorname{Hom}_{B, A}(N)}{\operatorname{ker} \varphi} .
$$

However, $(\varphi, \sigma) \in \operatorname{ker} \varphi$ if and only if $d_{\phi, \sigma}$ is inner. By Lemma $2, \operatorname{ker} \varphi=Z R_{A, B}(M) \times Z R_{B, A}(N)$. Thus, by this fact and (3), (2) holds.

Corollary 4 Let A and B be a commutative ring. By hypothesis of the above Theorem, we have $H^{1}(S, S) \cong H_{A, B}(M) \times$ $\operatorname{Hom}_{B, A}(N)$.

\section{Vanishing of the First Cohomology Group}

Let $X$ be a unitary $S$-bimodule, denote $X_{A A}=1_{A} X 1_{A}, X_{B B}=1_{B} X 1_{B}, X_{A B}=1_{A} X 1_{B}$ and $X_{B A}=1_{B} X 1_{A}$. For example, when $X=S$, we have $X_{A A}=A, X_{B B}=B, X_{A B}=M$ and $X_{B A}=N$. In this section, the relations between the first cohomology of $S$ with coefficients in $X$ and those of $A$ and $B$ with coefficients in $X_{A A}$ and $X_{B B}$, respectively, whenever $X_{A B}=0$, are investigated.

We started by illustrating the structure of derivations from a square algebra into its bimodules.

Let $\delta: S \longrightarrow X$ be a derivation. Then $\delta_{A}: A \longrightarrow X_{A A}$ defined by $\delta_{A}(a)=1_{A} \delta\left(\left[\begin{array}{ll}a & 0 \\ 0 & 0\end{array}\right]\right) 1_{A}$ and $\delta_{B}: B \longrightarrow X_{B B}$ defined by $\delta_{B}(b)=1_{B} \delta\left(\left[\begin{array}{ll}0 & 0 \\ 0 & b\end{array}\right]\right) 1_{B}$ are derivations. Moreover, the $\mathcal{R}$-linear maps $\tau: M \longrightarrow X_{A B}$, defined by $\tau(m)=1_{A} \delta\left(\left[\begin{array}{cc}0 & m \\ 0 & 0\end{array}\right]\right) 1_{B}$ and $\sigma: N \longrightarrow X_{B A}$ defined by $\sigma(n)=1_{B} \delta\left(\left[\begin{array}{ll}0 & 0 \\ n & 0\end{array}\right]\right) 1_{A}$ satisfy

(i) $\tau\left(\right.$ am) $=a \tau(m)+\delta_{A}(a) m$,

(ii) $\tau(m b)=\tau(m) b+m \delta_{B}(b)$,

(iii) $\sigma(n a)=\sigma(n) a+n \delta_{A}(a)$,

(iv) $\sigma(b n)=b \sigma(n)+n \delta_{B}(b) n$.

Conversely, if $\delta_{1}$ and $\delta_{2}$ are derivation from $A$ and $B$ into $X_{A A}$ and $X_{B B}$, respectively, and $\tau: M \longrightarrow X_{A B}$ and $\sigma: N \longrightarrow X_{B A}$ are any $\mathcal{R}$-linear maps satisfy in (i), (ii), (iii) and (iv), then the map $D\left(\left[\begin{array}{cc}a & m \\ n & b\end{array}\right]\right)=\delta_{1}(a)+\delta_{2}(b)+\tau(n)+\sigma(n)$ defines a derivation from $S$ into $X$. If $X_{A B}=0=X_{B A}$, then we may assume that $\tau$ and $\sigma$ are zero. Note that, in this case, $\delta_{A}(a) m=m \delta_{B}(b)=0=\delta_{B}(b) n=n \delta_{n}(a)$, for every $a \in A, b \in B, m \in M$ and $n \in N$. 
Now, we have the following:

Theorem 5 If $X_{A B}=0=X_{B A}$, where $X$ is a unitary $S$-module. Then

$$
H^{1}(S, X)=H^{1}\left(A, X_{A A}\right) \oplus H^{1}\left(B, X_{B B}\right)
$$

Proof. Suppose that $X_{A B}=0=X_{B A}$ and consider the $\mathcal{R}$-linear map $\rho: \operatorname{Der}(S, X) \longrightarrow H^{1}\left(A, X_{A A}\right) \oplus H^{1}\left(B, X_{B B}\right)$ defined by

$$
\delta \rightarrow\left(\delta_{A}+\operatorname{Inn}\left(A, X_{A A}\right), \delta_{B}+\operatorname{Inn}\left(B, X_{B B}\right)\right)
$$

If $\delta_{1} \in \operatorname{Der}\left(A, X_{A A}\right)$ and $\delta_{2} \in \operatorname{Der}\left(B, X_{B B}\right)$, then

$$
D\left(\left[\begin{array}{cc}
a & m \\
n & b
\end{array}\right]\right)=\delta_{1}(a)+\delta_{2}(b)
$$

is a derivation from $S$ into $X$ and

$$
\begin{aligned}
\rho(D) & =\left(\delta_{A}+N^{1}\left(A, X_{A A}\right), \delta_{B}+N^{1}\left(B, X_{B B}\right)\right) \\
& =\left(\delta_{1}+N^{1}\left(A, X_{A A}\right), \delta_{2}+N^{1}\left(B, X_{B B}\right)\right) .
\end{aligned}
$$

The last equation is deduced from the fact that $\delta_{A}(a)=1_{A}\left(\delta_{1}(a)+\delta_{2}(0)\right) 1_{A}=\delta_{1}(a)$ and $\delta_{B}(b)=1_{B}\left(\delta_{1}(0)+\delta_{2}(b)\right)$. Thus $\rho$ is surjective.

If $\delta \in \operatorname{ker} \rho$, then $\delta_{A} \in \operatorname{Inn}\left(A, X_{A A}\right)$ and $\delta_{B} \in \operatorname{Inn}\left(B, X_{B B}\right)$. Then $\delta_{A}(a)=a x-x a$ for some $x \in X_{A A}$ and $\delta_{B}(b)=b y-y b$ for some $y \in X_{B B}$. Then

$$
\begin{aligned}
& D\left(\left[\begin{array}{cc}
a & m \\
n & b
\end{array}\right]\right)=\delta_{A}(a)+\delta_{B}(b)=(a x-x a)+(b y-y b) \\
& =\left(\left[\begin{array}{cc}
a & m \\
n & b
\end{array}\right]\left[\begin{array}{cc}
1_{A} & 0 \\
0 & 0
\end{array}\right] x-x\left[\begin{array}{cc}
1_{A} & 0 \\
0 & 0
\end{array}\right]\left[\begin{array}{cc}
a & m \\
n & b
\end{array}\right]+x\left[\begin{array}{cc}
0 & m \\
n & 0
\end{array}\right]\right) \\
& +\left(\left[\begin{array}{cc}
a & m \\
n & b
\end{array}\right]\left[\begin{array}{cc}
0 & 0 \\
0 & 1_{B}
\end{array}\right] y-\left[\begin{array}{cc}
0 & m \\
n & 0
\end{array}\right] y-y\left[\begin{array}{cc}
0 & 0 \\
0 & 1_{B}
\end{array}\right]\left[\begin{array}{cc}
a & m \\
n & b
\end{array}\right]\right) \\
& =\left(\left[\begin{array}{cc}
a & m \\
n & b
\end{array}\right]\left[\begin{array}{cc}
1_{A} & 0 \\
0 & 0
\end{array}\right] x-x\left[\begin{array}{cc}
1_{A} & 0 \\
0 & 0
\end{array}\right]\left[\begin{array}{cc}
a & m \\
n & b
\end{array}\right]+x\left[\begin{array}{cc}
0 & m \\
0 & 0
\end{array}\right]-\left[\begin{array}{ll}
0 & 0 \\
n & 0
\end{array}\right] x\right) \\
& +\left(\left[\begin{array}{cc}
a & m \\
n & b
\end{array}\right]\left[\begin{array}{cc}
0 & 0 \\
0 & 1_{B}
\end{array}\right] y-\left[\begin{array}{cc}
0 & m \\
0 & 0
\end{array}\right] y-y\left[\begin{array}{cc}
0 & 0 \\
0 & 1_{B}
\end{array}\right]\left[\begin{array}{cc}
a & m \\
n & b
\end{array}\right]+y\left[\begin{array}{ll}
0 & 0 \\
n & 0
\end{array}\right]\right) \\
& =\left(\left[\begin{array}{cc}
a & m \\
n & b
\end{array}\right] x-x\left[\begin{array}{cc}
a & m \\
n & b
\end{array}\right]+\left[\begin{array}{cc}
1_{A} & 0 \\
0 & 0
\end{array}\right] x\left[\begin{array}{cc}
0 & m \\
0 & 0
\end{array}\right]\left[\begin{array}{cc}
0 & 0 \\
0 & 1_{B}
\end{array}\right]\right. \\
& \left.-\left[\begin{array}{cc}
0 & 0 \\
0 & 1_{B}
\end{array}\right]\left[\begin{array}{ll}
0 & 0 \\
n & 0
\end{array}\right] x\left[\begin{array}{cc}
1_{A} & 0 \\
0 & 0
\end{array}\right]\right) \\
& +\left(\left[\begin{array}{cc}
a & m \\
n & b
\end{array}\right] y-y\left[\begin{array}{cc}
a & m \\
n & b
\end{array}\right]-\left[\begin{array}{cc}
1_{A} & 0 \\
0 & 0
\end{array}\right]\left[\begin{array}{cc}
0 & m \\
0 & 0
\end{array}\right] y\left[\begin{array}{cc}
0 & 0 \\
0 & 1_{B}
\end{array}\right]\right. \\
& \left.+\left[\begin{array}{cc}
0 & 0 \\
0 & 1_{B}
\end{array}\right] y\left[\begin{array}{ll}
0 & 0 \\
n & 0
\end{array}\right]\left[\begin{array}{cc}
1_{A} & 0 \\
0 & 0
\end{array}\right]\right) \\
& =\left[\begin{array}{cc}
a & m \\
n & b
\end{array}\right](x+y)-(x+y)\left[\begin{array}{cc}
a & m \\
n & b
\end{array}\right]=\delta_{x+y}\left(\left[\begin{array}{cc}
a & m \\
n & b
\end{array}\right]\right) \text {. }
\end{aligned}
$$

Thus $D=\delta_{x+y}$. It is straightforward to show that

$$
\delta\left(\left[\begin{array}{ll}
a & 0 \\
0 & 0
\end{array}\right]\right)=1_{A} \delta\left(\left[\begin{array}{ll}
a & 0 \\
0 & 0
\end{array}\right]\right) 1_{A}-1_{B} \delta\left(\left[\begin{array}{cc}
1_{A} & 0 \\
0 & 0
\end{array}\right]\right) 1_{A}\left[\begin{array}{cc}
a & 0 \\
0 & 0
\end{array}\right] .
$$

Similarly,

$$
\delta\left(\left[\begin{array}{ll}
0 & 0 \\
0 & b
\end{array}\right]\right)=1_{B} \delta\left(\left[\begin{array}{ll}
0 & 0 \\
0 & b
\end{array}\right]\right) 1_{B}-\left[\begin{array}{ll}
0 & 0 \\
0 & b
\end{array}\right] 1_{B} \delta\left(\left[\begin{array}{cc}
1_{A} & 0 \\
0 & 0
\end{array}\right]\right) 1_{A}
$$


Also,

and

$$
\delta\left(\left[\begin{array}{cc}
0 & m \\
0 & 0
\end{array}\right]\right)=1_{B} \delta\left(\left[\begin{array}{cc}
0 & 0 \\
0 & b
\end{array}\right]\right) 1_{A}\left[\begin{array}{cc}
0 & m \\
0 & b
\end{array}\right]-\left[\begin{array}{cc}
a & m \\
0 & 0
\end{array}\right] 1_{B} \delta\left(\left[\begin{array}{cc}
1_{A} & 0 \\
0 & 0
\end{array}\right]\right) 1_{A}
$$

$$
\delta\left(\left[\begin{array}{ll}
0 & 0 \\
n & 0
\end{array}\right]\right)=1_{A} \delta\left(\left[\begin{array}{cc}
0 & 0 \\
0 & 1_{B}
\end{array}\right]\right) 1_{B}\left[\begin{array}{ll}
a & 0 \\
n & 0
\end{array}\right]-\left[\begin{array}{ll}
0 & 0 \\
n & b
\end{array}\right] 1_{A} \delta\left(\left[\begin{array}{cc}
0 & 0 \\
0 & 1_{B}
\end{array}\right]\right) 1_{B} .
$$

These follow that

$$
(\delta-D)\left(\left[\begin{array}{cc}
a & m \\
n & b
\end{array}\right]\right)=-\delta 1_{1_{B} \delta}\left(\left[\begin{array}{cc}
1_{A} & 0 \\
0 & 0
\end{array}\right]\right) \operatorname{1}_{A}\left(\left[\begin{array}{cc}
a & m \\
n & b
\end{array}\right]\right) .
$$

Therefore, we have $\delta-D \in \operatorname{Inn}(S, X)$, and so $\delta \in \operatorname{Inn}(S, X)$.

Conversely, let $\delta \in \operatorname{Inn}(S, X)$. Then there exists $x \in X$ such that

$$
\delta\left(\left[\begin{array}{cc}
a & m \\
n & b
\end{array}\right]\right)=\left[\begin{array}{cc}
a & m \\
n & b
\end{array}\right] x-x\left[\begin{array}{cc}
a & m \\
n & b
\end{array}\right] .
$$

So that

$$
\begin{aligned}
\delta_{A}(a) & =1_{A} \delta\left(\left[\begin{array}{ll}
a & 0 \\
0 & 0
\end{array}\right]\right) 1_{A}=1_{A}\left(\left[\begin{array}{ll}
a & 0 \\
0 & 0
\end{array}\right] x-x\left[\begin{array}{ll}
a & 0 \\
0 & 0
\end{array}\right]\right) 1_{A} \\
& =\left[\begin{array}{ll}
a & 0 \\
0 & 0
\end{array}\right] 1_{A}=1_{A}-1_{A} x 1_{A}\left[\begin{array}{ll}
a & 0 \\
0 & 0
\end{array}\right]=\delta_{1_{A} x 1_{A}}(a) .
\end{aligned}
$$

Similarly, $\delta_{B}(b)=\delta_{1_{B} x 1_{B}}(b)$. Hence $\delta_{A}$ and $\delta_{B}$ are inner and so $\delta \in \operatorname{ker} \rho$. Thus $\operatorname{Inn}(S, X)=\operatorname{ker} \rho$. We conclude that

$$
H^{1}(S, X)=\frac{\operatorname{Der}(S, X)}{\operatorname{Inn}(S, X)}=\frac{\operatorname{Der}(S, X)}{\operatorname{ker} \rho}=H^{1}\left(A, X_{A A}\right) \oplus H^{1}\left(B, X_{B B}\right)
$$

Corollary $6 H^{1}(S, M)=0=H^{1}(S, N)$.

Proof. With $X=M(X=N)$ we have

$$
H^{1}(S, M)=H^{1}(A, 0) \oplus H^{1}(B, 0) \quad\left(H^{1}(S, N)=H^{1}(A, 0) \oplus H^{1}(B, 0)\right)
$$

and this is zero.

Corollary $7 H^{1}(S, A)=0$ where

$$
S=\left[\begin{array}{cc}
A & A \\
A & A
\end{array}\right]=\left\{\left[\begin{array}{ll}
a & a \\
a & a
\end{array}\right] \mid a \in A\right\}
$$

Example 8 If $S=\left[\begin{array}{cc}\mathbb{Z} & \mathbb{Z}_{n} \\ \mathbb{Z}_{n} & \mathbb{Z}\end{array}\right]$ for $n>1$, then $H^{1}\left(S, \mathbb{Z}_{n}\right)=0$.

\section{Stability of the First Hochschild Cohomology}

Let $A$ and $\mathcal{R}$ be Banach algebras such that $A$ is a Banach $\mathcal{R}$-algebra with compatible actions, that is

$$
\alpha \cdot(a b)=(\alpha \cdot a) b,(a b) \cdot \alpha=a(b \cdot \alpha)
$$

for all $a, b \in A, \alpha \in \mathcal{R}$. Let $X$ be a Banach $A$-bimodule and a Banach $\mathcal{R}$-bimodule with compatible actions, that is

$$
\begin{aligned}
& \alpha \cdot(a \cdot x)=(\alpha \cdot a) \cdot x,(\alpha \cdot x) \cdot a=\alpha \cdot(x \cdot a) \\
& x \cdot(a \cdot \alpha)=(x \cdot a) \cdot \alpha,(a \cdot x) \cdot \alpha=a \cdot(x \cdot \alpha) \\
& a \cdot(\alpha \cdot x)=(a \cdot \alpha) \cdot x, x \cdot(\alpha \cdot a)=(x \cdot \alpha) \cdot a
\end{aligned}
$$


for all $a \in A, \alpha \in \mathcal{R}, x \in X$. Then we say that $X$ is a Banach $A$ - $R$-module. If moreover

$$
\alpha \cdot x=x \cdot \alpha \quad(\alpha \in \mathcal{R}, x \in X)
$$

then $X$ is called a commutative $A$ - $\mathcal{R}$-module.

Let $A$ and $B$ are Banach $\mathcal{R}$-algebras with units $1_{A}$ and $1_{B}$, respectively, $M$ is a Banach $\mathcal{R}$-bimodule, left Banach $A$-module and right Banach $B$-module $(A, B$-module) and $N$ is a Banach $\mathcal{R}$-bimodule, right Banach $A$-module and left $B$-module (B,A-module). Then $S=\left\{\left[\begin{array}{cc}a & m \\ n & b\end{array}\right] \mid a \in A, b \in B, m \in M, n \in N\right\}$ is a Banach $\mathcal{R}$-algebra equipped with the defined operations in section 1 and the following norm

$$
\left\|\left[\begin{array}{cc}
a & m \\
n & b
\end{array}\right]\right\|=\|a\|_{A}+\|b\|_{B}+\|m\|_{M}+\|n\|_{N} .
$$

Let $X$ be a unitary $S$-bimodule and $X_{A A}, X_{B B}, X_{A B}$ and $X_{B A}$ be similar to section 3. Assume that $X_{A B}=0=X_{B A}$. Let $\alpha \in \mathcal{R}$ and let $f_{1}, f_{2}, f_{3}: S \longrightarrow X$ be mappings. Define

$$
D_{\alpha}\left[f_{1}, f_{2}, f_{3}\right]\left(s_{1}, s_{2}\right)=f_{1}\left(\alpha s_{1}+\alpha s_{2}\right)-\alpha f_{2}\left(s_{1}\right)-\alpha f_{3}\left(s_{2}\right),
$$

and

$$
\delta\left[f_{1}, f_{2}, f_{3}\right]\left(s_{1}, s_{2}\right)=s_{1} f\left(s_{2}\right)-f\left(s_{1} s_{2}\right)+f_{3}\left(s_{1}\right) s_{2},
$$

for all $s_{1}, s_{2} \in S$. Similar to section 3, we obtain the mappings $f_{A}^{i}: A \longrightarrow X_{A A}$ and $f_{B}^{i}: B \longrightarrow X_{B B}$ for $i=1,2,3$ that are defined as

$$
f_{A}^{i}(a)=e_{A} f_{i}\left(\left[\begin{array}{ll}
a & 0 \\
0 & 0
\end{array}\right]\right) e_{A} \text { and } f_{B}^{i}(b)=e_{B} f_{i}\left(\left[\begin{array}{ll}
0 & 0 \\
0 & b
\end{array}\right]\right) e_{B},
$$

for all $a \in A$ and $b \in B$.

Theorem 9 Let $\lambda, \gamma \in \mathbb{R}^{+}$and $f_{1}, f_{2}, f_{3}: S \longrightarrow X$ be mappings that satisfy

$$
\begin{gathered}
\left\|D_{\alpha}\left[f_{1}, f_{2}, f_{3}\right]\left(s_{1}, s_{2}\right)\right\| \leq \lambda, \\
\left\|\delta\left[f_{1}, f_{2}, f_{3}\right]\left(s_{1}, s_{2}\right)\right\| \leq \gamma .
\end{gathered}
$$

If for any $s_{i}=0, i=1,2$, we have $f_{i}\left(s_{i}\right)=0$, then there exists a unique inner derivation $D$ such that

$$
\begin{aligned}
\left\|f_{1}(s)-D(s)\right\| & \leq 6 \lambda, \\
\left\|f_{2}(s)-D(s)\right\| & \leq 12 \lambda, \\
\left\|f_{3}(s)-D(s)\right\| & \leq 12 \lambda,
\end{aligned}
$$

for all $s \in S$.

Proof. Let $\alpha=1_{\mathcal{R}}$ (unit of $\mathcal{R}$ ) and $s_{2}=0$, then

$$
\left\|f_{1}\left(s_{1}\right)-f_{2}\left(s_{1}\right)\right\| \leq \lambda,
$$

for all $s_{1} \in S$. Similarly,

$$
\left\|f_{1}\left(s_{2}\right)-f_{3}\left(s_{2}\right)\right\| \leq \lambda,
$$

for all $s_{2} \in S$. By repeating the above stated relations we obtain the desire.

\section{Acknowledgements}

Collate acknowledgements in a separate section at the end of the article before the references. List here those individuals who provided help during the research (e.g., providing language help, writing assistance or proof reading the article, etc.).

\section{References}

Brodmann, M. P., \& Sharp, R. Y. (1998). Local cohomology: an algebraic introduction with geometric applications. Cambridge Studies in Advanced Mathematics, 60. Cambridge University Press, Cambridge.

https://doi.org/10.1017/CBO9780511629204 
Buchweitz, R. E. (2003). Morita contexts, idempotents, and Hochschild cohomology with applications to invariant rings. Contemp. Math., 331, 25-53. https://doi.org/10.1090/conm/331/05901

Cheung, W. S. (2001). Commuting maps of triangular algebras, J. London Math. Soc., 63(1), 117-127. https://doi.org/10.1112/S0024610700001642

Cheung, W. S. (2003). Lie derivations of triangular algebras, Linear Multilinear Algebra, 51(3), 299-310. https://doi.org/10.1080/0308108031000096993

Christensen, E. (1977). Derivations of nest algebras, Math. Ann., 229, 155-161. https://doi.org/10.1007/BF01351601

Coelho, S. P., \& Milies, C. P. (1993). Derivations of upper triangular matrix rings. Linear Algebra Appl., 187, $263-267$. https://doi.org/10.1016/0024-3795(93)90141-A

Donsig, A. P., Forrest, B. E., \& Marcoux, L. W. (1996). On derivations of semi-nest algebras. Houston J. Math., 22(2), 375-398.

Du, Y., \& Wang, Y. (2012). Lie derivations of generalized matrix algebras. Linear Algebra Appl., 437, 2719-2726. https://doi.org/10.1016/j.laa.2012.06.013

Huneke, C. (1992). Problems on Local cohomology, in: Free Resolution in Commutative Algebra and Algebraic Geometry, (Sundance, Utah, 1990) Mathematics, 2, Jones and Barlett, pp.93-108. Research Notes in Mathematics, 2, Jones and Barlett Publisher, Boston, MA.

Ji, P., \& Qi, W. (2011). Characterizations of Lie derivations of triangular algebras. Linear Algebra Appl., 435, 1137-1146. https://doi.org/10.1016/j.laa.2011.02.048

Li, Y. B., \& Wei, F. (2012). Semi-centralizing maps of generalized matrix algebras. Linear Algebra Appl., 436, 11221153. https://doi.org/10.1016/j.laa.2011.07.014

Li, Y. B., \& Xiao, Z. K. (2011). Additivity of maps on generalized matrix algebras. Electron. J. Linear Algebra, 22, 743-757. https://doi.org/10.13001/1081-3810.1471

Rotman, J. (2009). An introduction to homological algebra, Second edition, New York, Speringer-Verlag. https://doi.org/10.1007/b98977

Xiao, Z. K., \& Wei, F. (2010). Commuting mappings of generalized matrix algebras. Linear Algebra Appl, 433, 21782197. https://doi.org/10.1016/j.laa.2010.08.002

\section{Copyrights}

Copyright for this article is retained by the author(s), with first publication rights granted to the journal.

This is an open-access article distributed under the terms and conditions of the Creative Commons Attribution license (http://creativecommons.org/licenses/by/4.0/). 